\title{
Assessment of the Ability of Different Cleaning Protocols to Remove Eugenol-based Endodontic Sealer from the Root Dentin
}

\author{
Ana P Farina ${ }^{1}$, Emanuele de Oliveira ${ }^{2}$, Alana Disarz ${ }^{3}$, Ana LC de Moura ${ }^{4}$, Migueli Durigon ${ }^{5}$, Matheus A Souza ${ }^{6}$, Doglas Cecchin ${ }^{7}$
}

\begin{abstract}
Aim: The aim of this study is to assess three protocols for removing a eugenol-based filling endodontic sealer from root dentin and the bond strength of fiberglass posts luted with resin cement.

Materials and methods: Sixty single-rooted bovine teeth were prepared and filled with the Endofill endodontic sealer and gutta-percha cones. After 7 days, $9 \mathrm{~mm}$ of the filling was removed and divided into five groups $(n=12)$ according to the cleaning protocol: $\mathrm{G} 1$ (negative control), unfilled; G2 (positive control), saline solution; G3, 95\% alcohol; G4, amyl acetate; and G5, Largo bur. The canals were hybridized and fiberglass posts were luted with resin cement. They were cross-sectioned in slices of $1 \mathrm{~mm}$ and subjected to the push-out test. The other samples were sectioned longitudinally and visualized in a scanning electron microscope (SEM), and the existing chemical elements were quantified by energy dispersive spectroscopy (EDS). The statistical analysis used variance analysis (ANOVA)-one way and Fisher's test, at a 5\% significance level.

Results: The $95 \%$ alcohol was more effective for cleaning the canal, resulting in a clean dentinal surface and bond strength statistically similar to the negative control. The Largo bur was also statistically similar to the negative control, with only a few debris impregnated on the wall. Amyl acetate showed more sealer residues on the canal walls, with a consequent lower bond strength value than the other groups, only higher than the positive control.
\end{abstract}

Conclusion: The $95 \%$ alcohol and Largo burs may be used after removing the canal filling, so that the bond strength is improved when using the eugenol-based sealer.

Keywords: 95\% alcohol, Adhesion, Amyl acetate, Fiberglass posts, Largo bur, Zinc oxide-eugenol sealer.

The Journal of Contemporary Dental Practice (2019): 10.5005/jp-journals-10024-2575

\section{INTRODUCTION}

The restoration of endodontically treated teeth is a challenging practice for most dentists, because of the great loss of the crown structure that usually occurs. Considering the need for rehabilitation, the intraradicular posts appeared as a form of retention to the crown restoration. ${ }^{1,2}$

Cast metal cores are traditionally used despite having some limitations, such as high elasticity modulus, which increases the potential for irreparable fractures in tooth remnants. ${ }^{3,4}$ However, fiberglass posts allowed great advances, especially regarding the mechanical properties. Their high flexural strength and elastic modulus similar to dentin minimize the stress transfer to the root walls, decreasing the occurrence of fractures..$^{5,6}$

When using fiberglass posts, good adhesion to the structures of root dentin is required. However, in some endodontic filling sealers, eugenol interferes negatively with the adhesion of fiberglass posts to root dentin. ${ }^{7,8}$ This occurs because a canal filled with a eugenol-based sealer, even with proper canal preparation for post cementation, still presents residues retained on the dentinal walls and inside the dentinal tubules, which inhibit the full polymerization of the resin cement. ${ }^{9,10}$ Although endodontists still make extensive use of zinc oxide-eugenol endodontic sealers, ${ }^{11,12}$ it is important to resort to removal alternatives to obtain an adequate biomechanical performance of the final restoration. ${ }^{13}$

Therefore, this study aimed to assess the effectiveness of three protocols for removing the eugenol-based filling endodontic sealer from the root dentin. The null hypothesis is that the application of such techniques promotes adequate cleaning of the canals.
${ }^{1}$ Department of Restorative Dentistry, Faculty of Dentistry, University of Passo Fundo, UPF, Passo Fundo, Rio Grande do Sul, Brazil

${ }^{2-4}$ Faculty of Dentistry, University of Passo Fundo, UPF, Passo Fundo, Rio Grande do Sul, Brazil

${ }^{5}$ University of Passo Fundo, UPF, Passo Fundo, Rio Grande do Sul, Brazil

${ }^{6,7}$ Department of Endodontics, Faculty of Dentistry, University of Passo Fundo, UPF, Passo Fundo, Rio Grande do Sul, Brazil

Corresponding Author: Doglas Cecchin, University of Passo Fundo, Campus I, School of Dentistry, BR 285, Km 171, Bairro São José, Passo Fundo, Rio Grande do Sul, Brazil, Phone: +55 543316 8402, e-mail: doglas@upf.br

How to cite this article: Farina AP, de Oliveira $E$, et al. Assessment of the Ability of Different Cleaning Protocols to Remove Eugenolbased Endodontic Sealer from the Root Dentin. J Contemp Dent Pract 2019;20(6):657-663.

Source of support: Nil

Conflict of interest: None

\section{Materials and Methods}

\section{Sample Collection and Preparation}

Sixty freshly extracted bovine incisors were used. The teeth were cleaned with periodontal curettes and frozen until the moment of use. Lines at $15 \mathrm{~mm}$ from the apex were traced with a digital caliper and an overhead projector pen. Next, the teeth were sectioned with a double-sided diamond disc (KG Sorensen, Barueri, SP, Brazil), separating the root portion of interest. The remaining pulp tissue

(0) The Author(s). 2019 Open Access This article is distributed under the terms of the Creative Commons Attribution 4.0 International License (https://creativecommons. org/licenses/by-nc/4.0/), which permits unrestricted use, distribution, and non-commercial reproduction in any medium, provided you give appropriate credit to the original author(s) and the source, provide a link to the Creative Commons license, and indicate if changes were made. The Creative Commons Public Domain Dedication waiver (http://creativecommons.org/publicdomain/zero/1.0/) applies to the data made available in this article, unless otherwise stated. 
was removed with hand K-files (Dentsply Maillefer, Petrópolis, RJ, Brazil), whose diameter matched the canal.

The canal was prepared with burs for postpreparation (Angelus, Londrina, PR, Brazil). As auxiliary chemical substance, $2 \%$ chlorhexidine was used followed by irrigation with $5 \mathrm{~mL}$ of saline solution at every instrument change. Lastly, all samples were irrigated with $2 \mathrm{~mL}$ of $17 \%$ ethylenediaminetetraacetic acid (EDTA) for 3 minutes, agitating the substance inside the canal with a hand file, and there was a final irrigation of additional $5 \mathrm{~mL}$ of saline solution.

The canals were dried with an absorbent paper cone and filled with the Endofill endodontic sealer (Dentsply Maillefer) and guttapercha cones (Odous de Deus, Biodental, SC, Brazil). The sealer was manipulated until the consistency of a fillet between the plate and the elevated spatula was reached, and it was inserted into the canal with a Lentulo bur. The cones were cut with a heated Paiva clamp (Golgran, São Caetano do Sul, SP, Brazil) and the samples were stored in Eppendorfs at 100\% humidity, for 7 days.

For intraradicular preparation, two-third of the canal filling was removed, maintaining $5 \mathrm{~mm}$ of filling. Removal started with heating a \#1 Rhein probe (Golgran, São Caetano do Sul, SP, Brazil) followed by the use of a low-rotation bur for postpreparation. A single operator performed all the steps of the sample preparation process to minimize the research bias potential.

\section{Division of Experimental Groups}

The roots were divided into five groups $(n=12)$ according to the canal cleaning protocol: G1-unfilled (negative control); G2 - saline solution (positive control); G3-95\% alcohol; G4-amyl acetate; and G5-Largo bur, used only until the wall was visibly clean.

The substances studied were pharmaceutically manipulated before the moment of use. The dentinal surface was cleaned with a cotton swab adapted to a \#40 file, soaked in the cleaning substance, and rubbed against the canal walls for 3 minutes.

\section{Scanning Electron Microscopy (SEM) and Energy Dispersive Spectroscopy (EDS)}

Two roots from each group were analyzed by SEM (VEGA LM 3 Tescan, São Bernardo do Campo, SP, Brazil) to verify the level of dentinal cleanliness obtained. Two longitudinal grooves were produced in the outer surface of each root, which were later cleaved with the help of a chisel, resulting in two sections per specimen. Then, they were dehydrated in solutions with gradually increasing alcohol concentrations of $30,50,70,90$, and $100 \%$, with 30 minutes of immersion in each substance. Lastly, the specimens were immersed in acetone for 5 minutes and stored in a $50^{\circ} \mathrm{C}$ stove for 10 days. $^{14}$

The specimens were attached in aluminum-copper alloy stubs with a double-sided carbon tape and sputter coated with a layer of gold at a thickness of $5 \mathrm{~nm}$, using a Q150RES metallizer (Quorum, Laughton, East Sussex). Next, they were analyzed using an SEM operating at $5 \mathrm{kV}$ and a qualitative analysis was performed on the level of dentinal cleanliness obtained. The samples were analyzed initially with a magnification of $1000 \times$, followed by $2000 \times$ in the middle third, in which the images representing each group were obtained. Three different measurements were taken for each root and the mean value was calculated.

After capturing the SEM image, the EDS system was mapped to verify what chemical elements were present in the sample. The value of atomic percentage for each specimen was recorded and the resulting mean value was accepted as a representative image of the root canal area. The number of elements found in dentin was analyzed, such as calcium (Ca), phosphorus (P), and magnesium (Mg). The presence and the number of other elements potentially from the Endofill sealer were also verified.

According to the manufacturer's information, the sealer consists of zinc oxide, barium sulfate, sodium borate, bismuth subcarbonate, hydrogenated resin, eugenol, sweet almond oil, and BHT. Therefore, it verified the presence of elements, such as zinc $(\mathrm{Zn})$ and barium $(\mathrm{Ba})$, which indicate the permanence of sealer residues in the dentinal wall. EDS was used for additional analysis, adding information to the SEM results.

\section{Preparation, Molding, and Cementation of Fiberglass Posts}

The dentin of the remaining samples $(n=10)$ was etched with $37 \%$ phosphoric acid (FGM, Joinville, SC, Brazil) for 15 seconds, washed with $5 \mathrm{~mL}$ of distilled water, the canal was dried with an endodontic suction tube, and the ScotchBond Multipurpose ${ }^{\mathrm{TM}}$ adhesive system (3M ESPE, St. Paul, MN, USA) was applied according to the manufacturer's instructions.

The fiberglass posts (Angelus, Londrina, PR, Brazil) were prepared with the application of $37 \%$ phosphoric acid on their surface for 20 seconds, washed with water, dried with air blasts, and the ScotchBond Multipurpose adhesive system was applied and photoactivated for 40 seconds per buccal and palatal surface.

For molding the post, the root canal was filled with Natrosol gel (Natupharma, Passo Fundo, RS, Brazil), the fiberglass posts were surrounded by a composite resin (Opallis ${ }^{\mathrm{TM}}$ enamel A1, FGM, Brazil), and the set was inserted into the root canal. It was photoactivated in position for 4 seconds and the buccal region of the post and the tooth were marked for future cementation in the same position. The relined post was removed from inside the root canal and the polymerization of the composite resin was finished, with activation for 40 seconds per buccal and palatal surface.

The canal was irrigated with $5 \mathrm{~mL}$ of distilled water to remove the lubricant material, dried with absorbent paper points, and a thin bond layer was reapplied, removing the excess. The canal was photopolymerized for additional 40 seconds. The molded post was slightly worn with a \#11 scalpel blade (Solidor, Hebei Med e Health, China) for improved canal adaptation.

For cementation, the dual Rely $X \mathrm{ARC}^{\mathrm{TM}}$ resin cement (3M ESPE, USA) was used. It was manipulated according to the manufacturer's instructions and inserted in the canal aided by the syringes of the Centrix system and Accudose needle (DFL, Rio de Janeiro, RJ, Brazil). The post was placed inside the root canal, stabilized manually for 20 seconds, and photopolymerized for 5 seconds. The excess sealer was removed and light curing was performed for 40 seconds per surface (buccal, lingual, mesial, and distal). The post was stored at $100 \%$ humidity for 24 hours.

\section{Push-out Test}

The roots were cross-sectioned with the help of a precision cutter (Minitom, Struers A/S, Ballerup, Denmark) at $300 \mathrm{rpm}$ under constant refrigeration, resulting in one slice from each third of the canal, with approximately $1 \mathrm{~mm}$ of thickness each. These slices were placed in the testing machine and subjected to the push-out test (EMIC DL2000, São José dos Pinhais, PR, Brazil) with a load of 200 $\mathrm{N}$ at $0.5 \mathrm{~mm} /$ minute, from the apex to the crown. 
To calculate the bond strength in Mega Pascal (MPa), the load obtained in Newton (N) was divided by the bonding area at the moment of displacement, in millimeters $(\mathrm{mm})$. The bonding area was found by the truncated cone formula:

$\pi(R+r)\left[h^{2}+(R-r)^{2}\right]^{0.5}$

In the formula above, " $\pi$ " represents the constant 3.1416; " $R$ " represents the largest radius of the root canal in the cervical portion of the disc; and " $r$ " represents the smallest radius of the root canal in the apical portion of the disc. These values were measured with the help of the Imagelab 2.3 software (Borra \& Pizzarro, São Paulo, Brazil). Additionally, " $h$ ", which represents the height value of the disc, was measured with a digital caliper (Vonder Paquímetro Eletrônico Digital, Curitiba, PR, Brazil).

The bond strength results (in $\mathrm{MPa}$ ) were tabulated and subjected to ANOVA. For multiple comparisons, Fisher's test was used at a $5 \%$ significance level.

\section{Failure Mode Analysis}

All fractured specimens were observed in a stereoscopic magnifying glass with a magnification of $20 \times$ (Lambda Let 2, ATTO Instruments Co., Hong Kong, China) in both directions of the disc (cervical and apical) to determine the type of fracture affecting the sample. The fracture mode was classified, according to Cecchin et al., ${ }^{7}$ into five types: (1) adhesive, between the post and the resin cement (no cement visible around the post); (2) mixed, with resin cement covering $0-50 \%$ of the total post-diameter; (3) mixed, with resin cement covering $50-100 \%$ of the post-surface; (4) adhesive, between resin cement and root dentin (post surrounded by resin cement); and (5) cohesive, in dentin.

\section{Results}

Table 1 shows the bond strength values obtained and their failure modes, according to each dentinal cleaning protocol applied.

The best bond strength values were obtained in the negative control group. From the cleaning protocols tested, 95\% alcohol and the use of Largo bur were the most effective, because they resulted in values statistically similar to the negative control group ( $p>0.05$ ). Amyl acetate presented results statistically similar to $95 \%$ alcohol and the Largo bur ( $p>0.05)$, but not to the negative control $(p<0.05)$. The worst values were found when the root dentin was cleaned with saline solution.

The fracture analysis showed the prevalence of failure types 2,3 , and 4 for the positive control group (saline solution). The negative control group (without endodontic treatment) showed the prevalence of failure types 1 and 2 . For $95 \%$ alcohol, most failures were mixed ( $0-50 \%)$ and cohesive in dentin ( 2 and 5 , respectively), for amyl acetate, the failures were mixed (0-50\%) and adhesive in cement/dentin ( 2 and 4, respectively), while the group that used Largo burs presented mixed failures ( $0-50 \%)$ and cohesive in dentin ( 2 and 5, respectively).

In SEM, the negative control group presented a clean dentinal surface, with few debris particles from the smear layer produced during root canal preparation (Fig. 1A). The positive control group showed a dentinal surface with a dense smear layer buffering the dentinal tubules (Fig. 1B). Moreover, after EDS mapping, the positive control group presented a higher number of sealer elements, such as zinc and barium (1.6\% and 3.6\%, respectively) (Fig. 2B), which were undetected previously in the EDS of the negative control group (Fig. 2A).

The $95 \%$ alcohol and Largo bur groups were effective in cleaning the dentinal wall, leaving it with the lowest amount of sealer residues (Figs $1 \mathrm{C}$ and $\mathrm{E}$, respectively). The cleaning protocol that got $95 \%$ alcohol was similar to negative control. In EDS, both groups presented low rates of sealer elements. The alcohol group had $1.8 \%$ zinc and no barium in dentin composition, while the bur group had $1 \%$ barium and $0.8 \%$ zinc, which confirms the removal, but maintaining the rates of dentinal components stable (Figs $2 \mathrm{C}$ and $E$, respectively). The group in which cleaning was performed with amyl acetate showed inferior behavior (Fig. 1D), because it removed a certain amount of sealer from the dentinal walls, but not as effectively as the other groups, maintaining most of the obliterated dentinal tubules. In EDS, such group presented high rates of barium and zinc remnants ( $2.2 \%$ and $3.1 \%$, respectively), similar to the positive control (Fig. 2D).

\section{Discussion}

The use of intraradicular posts in endodontically treated teeth with great crown loss is a rather common restorative procedure, aiming to improve the retention of the crown restoration. The cast metal cores are still extensively used, but the intense search for aesthetics turned the use of fiberglass posts increasingly frequent, especially in the anterior region, considering that metal posts may suffer corrosion and cause marginal discoloration. ${ }^{2,15}$ Moreover, there are other advantages to fiberglass posts, such as the simplification of the restoration procedure by eliminating laboratory steps, the ease of removal when retreatment is required, and the preservation of tooth structure with less canal preparation, minimizing the weakening of the remaining root. ${ }^{2,16}$

Current studies on post-systems have focused on their mechanical properties, showing that posts with elasticity modulus closer to dentin, such as fiberglass posts, prevent stress concentration along the remaining root. ${ }^{4,7,8}$ In addition, a meta-

Table 1: Bond strength means $(\mathrm{MPa})$ and their respective standard deviations $( \pm)$ and fracture pattern, according to the treatments

\begin{tabular}{|c|c|c|c|c|c|c|}
\hline \multirow[b]{2}{*}{ Groups } & \multirow[b]{2}{*}{$\begin{array}{l}\text { Bond strength } \\
\text { (mean } \pm S D)\end{array}$} & \multicolumn{5}{|l|}{ Fracture mode } \\
\hline & & $\begin{array}{l}\text { Type } 1 \text { adhesive } \\
\text { (post-cement) }\end{array}$ & $\begin{array}{l}\text { Type } 2 \text { mixed } \\
(0-50 \%)\end{array}$ & $\begin{array}{l}\text { Type } 3 \text { mixed } \\
(50-100 \%)\end{array}$ & $\begin{array}{l}\text { Type } 4 \text { adhesive } \\
\text { (cement-dentin) }\end{array}$ & $\begin{array}{l}\text { Type } 5 \text { cohesive } \\
\text { in dentin }\end{array}$ \\
\hline $\begin{array}{l}\text { No endodontic treatment } \\
\text { (negative control) }\end{array}$ & $6.73( \pm 2.33)^{\mathrm{a}}$ & 11 & 11 & 4 & 0 & 4 \\
\hline Saline solution (positive control) & $1.69( \pm 1.83)^{c}$ & 3 & 5 & 9 & 10 & 3 \\
\hline $95 \%$ alcohol & $5.69( \pm 1.92)^{a, b}$ & 1 & 10 & 2 & 7 & 10 \\
\hline Amyl acetate & $3.92( \pm 2.15)^{b, c}$ & 2 & 10 & 4 & 9 & 5 \\
\hline Largo bur & $5.96( \pm 2.13)^{a, b}$ & 1 & 10 & 5 & 4 & 10 \\
\hline
\end{tabular}

Means followed by the same letters are statistically equal. Equal letters do not differ statistically from each other $(p>0.05)$ 

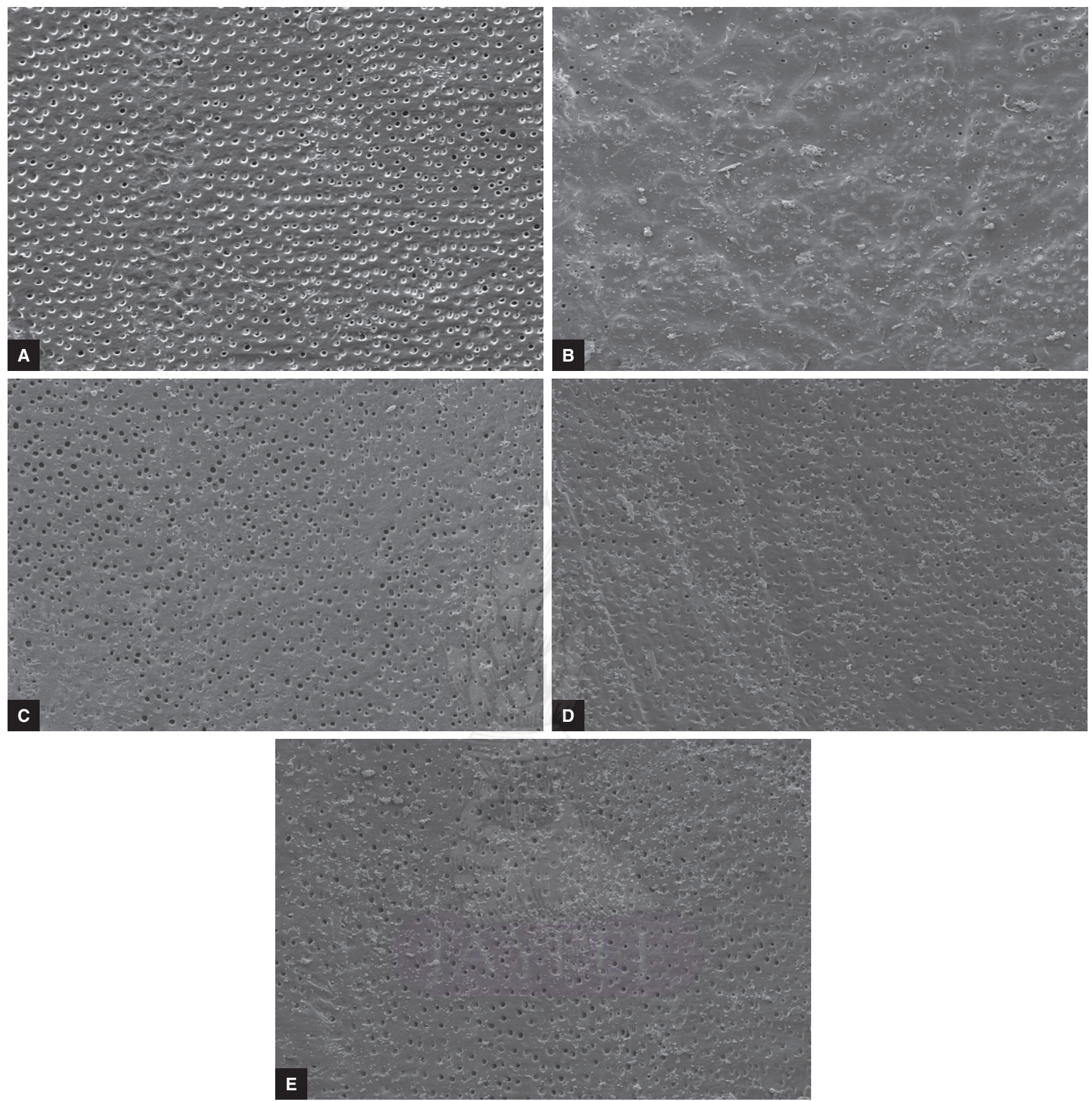

Figs 1 A to E: SEM images of the patterns of groups: (A) Negative control; (B) Positive control; (C) 95\% alcohol; (D) Amyl acetate; and (E) Largo bur; with a magnification of $2000 x$

analysis published by Zhou and Wang ${ }^{17}$ shows that teeth with metal posts present more catastrophic failures, which is compatible with horizontal root fractures in the middle third or longitudinal fractures. This is because of the greater toughness of such material. However, when failures occur with fiber posts, they are considered repairable, limited to the cervical third or the crown portion. ${ }^{4}$

Zinc oxide-eugenol sealers have been used in dentistry for many years to fill canals in the orthodontic treatment and they are still extensively used because of their satisfactory properties and long clinical history of success. ${ }^{11,12,15}$ However, the eugenol residues of these sealers that remain bonded to the dentinal walls interact with the monomeric free radicals and consequently prevent the adequate polymerization of the resin cement. ${ }^{18}$ Moreover, such sealers leave an oily layer of debris that is hard to remove, and its permanence results in low penetration of adhesives in dentin, also affecting adhesion. ${ }^{19}$

To minimize the effects of eugenol, proper cleaning procedures should be applied before cementing the posts. There is still no consensus in the literature regarding the best protocol to be used, but some procedures for cleaning the filling material have been described, such as the use of endodontic hand files, rotary instruments, heat-carrying devices, or ultrasonic instruments. ${ }^{20}$ 

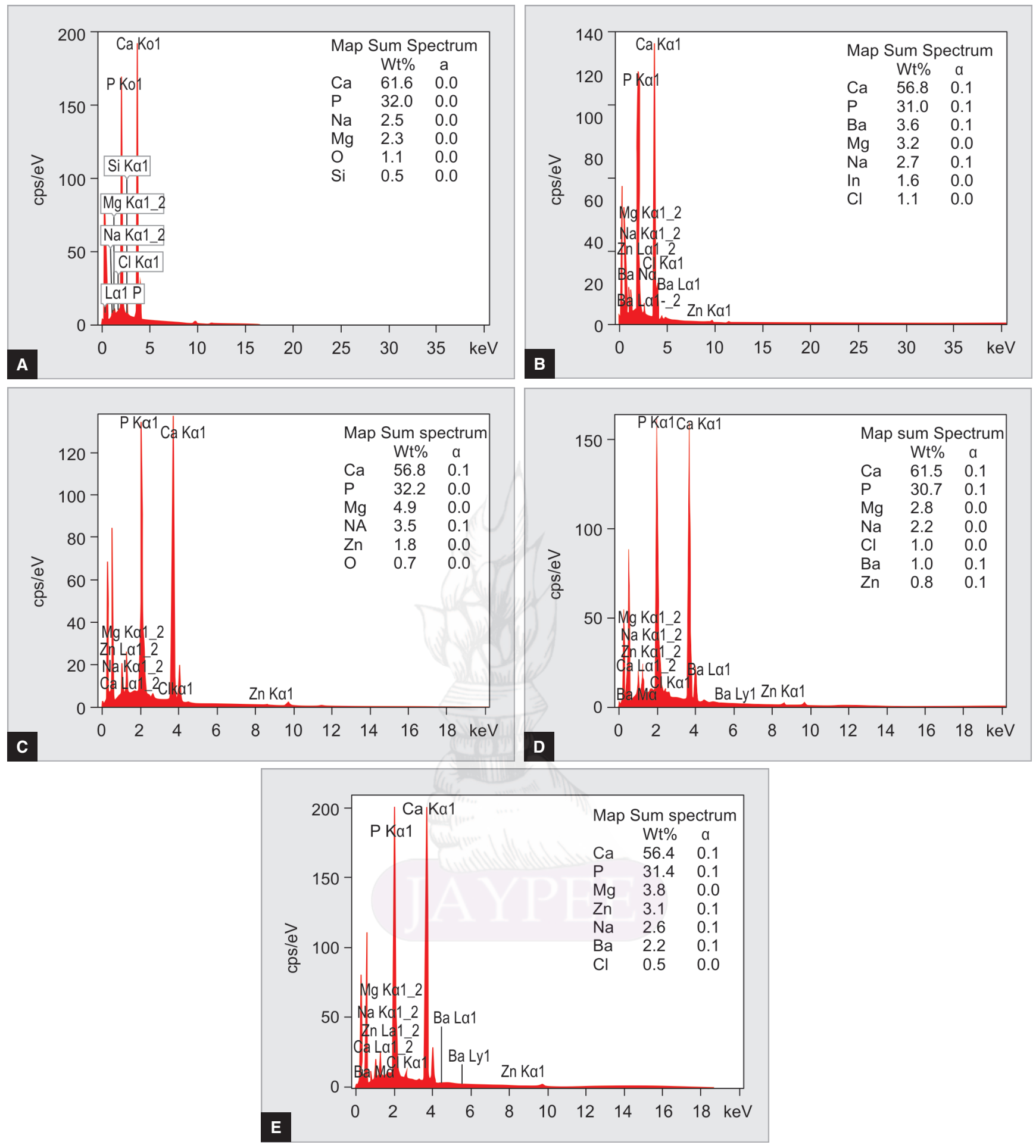

Figs 2A to E: Images of the values obtained in EDS for groups: (A) Negative control; (B) Positive control; (C) 95\% alcohol; (D) Amyl acetate; and (E) Largo bur

Another alternative is the combination of a given technique with solvents, which dissolve the filling material inside the canal. ${ }^{20,21}$

The results obtained show that the root canals cleaned with 95\% alcohol and the use of Largo burs provided good bond strength between the fiberglass post and the root dentin, and they are statistically similar to the negative control. The unfilled group showed failures that evidence a better bonding quality in the interface between resin cement and dentin, probably because of the complete absence of the eugenol-based sealer. In the other groups, the highest number of failures were mixed (0-50\%), in which more than $50 \%$ of the sealer remained bonded to dentin, in addition to a great number of cohesive fractures in dentin, showing a good bond strength of the resin cement/dentin set. The assessment of SEM images and EDS values confirm such affirmation, showing an 
adequate cleaning of the dentinal surface. The $95 \%$ alcohol was able to remove the eugenol-based sealer from the root dentin more efficiently. The SEM images showed a dentin with open dentinal tubules.

In a study by Altmann et al., ${ }^{22}$ the authors affirm that the procedures performed during the preparation of the postspace, such as the use of burs or cleaning with alcohol, are not effective. However, the results obtained in this study show the effectiveness of cleaning the root dentin with alcohol, agreeing with the findings by Bronzato et al., ${ }^{23}$ who also assessed the dentinal cleaning of eugenol-based sealer and obtained good results with alcohol. This is because NiTi rotary instruments and Largo burs have a specific anatomy and a cross-section that favor their use. Hence, when used to remove the filling material inside the canal, they work by expelling the filling material out of the canal, facilitating its removal. ${ }^{24}$ This justifies the findings of this study, in which SEM images show the dentinal tubules unobliterated, but with the presence of debris on the dentinal surface due to the cut produced by the instrument, and low rates of sealer elements detected by EDS.

On the contrary, amyl acetate is a solvent that works well on resins. ${ }^{25}$ However, this solvent overly softens the filling material and ends up leading gutta-percha and sealer residues into the dentinal tubules, later complicating their removal. ${ }^{21}$ The SEM images showed the dentinal tubules obliterated with sealer residues. As for the failure mode, there was a higher number of mixed $(0-50 \%)$ and adhesive failures between cement and dentin, showing bonding deficiency. The EDS showed that amyl acetate presented more permanence of eugenol-based sealer residues in the dentinal tubules, rejecting the hypothesis of the study.

Saline solution was not effective for removing eugenol remnants, so it presented the dentinal wall impregnated with the highest amount of debris and residues of the endodontic filling sealer, as well as obliterated dentinal tubules, when compared to the other groups. Additionally, the lowest bond strength values were found in the samples of this group. As for fracture analysis, the types of failures prevalent in the positive control group (saline solution) characterize poor adhesion between resin cement and dentin. This is because eugenol is almost insoluble in water and saline solution, dissolving only in alcohol, chloroform, ether, and other oils. This may be verified by the images presented in the EDS, confirming the presence of higher rates of elements from the filling sealer in the dentinal walls.

\section{ConCLUSION}

The use of $95 \%$ alcohol after removing root canal fillings is essential for removing zinc oxide-eugenol sealers, because it is the only solution capable of cleaning and unobliterating the dentinal tubules, favoring the adhesion of fiber posts to the root dentin.

\section{Clinical Significance}

Zinc and barium present in the eugenol root canal sealer inhibit the adhesive system polymerization during the cementation of the fiberglass post, indicating that Largo bur and 95\% alcohol improve the bond strength.

\section{ACKNOWLEDgement}

The authors would like to thank Angelus Inc. for donating the fiber posts. This investigation was supported by the Foundation for
Research Support of the State of Rio Grande do Sul (Fundação de Amparo à Pesquisa do Estado do Rio Grande do Sul_FAPERGS).

\section{References}

1. Toman M, Toksavul S, et al. The evaluation of displacement resistance of glass FRC posts to root dentine using a thin slice push-out test. Int Endod J 2009;42(9):802-810. DOI: 10.1111/j.13652591.2009.01586.x.

2. Choi Y, Pae A, et al. The effect of surface treatment of fiber-reinforced posts on adhesion of a resin-based luting agent. J Prosthet Dent 2010;103(6):362-368. DOI: 10.1016/S0022-3913(10)60078-9.

3. Lassila LVJ, Tanner J, et al. Flexural properties of fiber reinforced root canal posts. Dent Mater 2004;20(1):29-36.

4. Barcellos RR, Correia DPD, et al. Fracture resistance of endodontically treated teeth restored with intra-radicular post: the effects of post system and dentine thickness. J Biomech 2013;46(15):2572-2577. DOI: 10.1016/j.jbiomech.2013.08.016.

5. Schwartz RS, Robbins JW. Post placement and restoration of endodontically treated teeth: a literature review. J Endod 2004;30(5):289-301. DOI: 10.1097/00004770-200405000-00001.

6. Asmussen E, Peutzfeldt A, et al. Finite element analysis of stresses in endodontically treated, dowel-restored teeth. J Prosthet Dent 2005;94(4):321-329. DOI: 10.1016/j.prosdent.2005.07.003.

7. Cecchin $D$, Farina AP, et al. Effect of root canal sealers on bond strength of fiberglass posts cemented with self-adhesive resin cements. Int Endod J 2011;44(4):314-320. DOI: 10.1111/j.1365-2591.2010.01831.x.

8. Cecchin D, Farina AP, et al. Effect of root-canal sealer on the bond strength of fiberglass post to root dentin. Acta Odontol Scand 2011;69(1):95-100. DOI: 10.3109/00016357.2010.536908.

9. Teixeira CS, Pasternak-Junior B, et al. Influence of endodontic sealers on the bond strength of carbon fiber posts. J Biomed Mater Res 2008;84(2):430-435. DOI: 10.1002/jbm.b.30888.

10. Aleisa $K$, Alghabban R, et al. Effect of three endodontic sealers on the bond strength of prefabricated fiber posts luted with three resin cements. J Prosthet Dent 2012;107(5):322-326. DOI: 10.1016/S00223913(12)60084-5.

11. Mosharraf R, Zare S. Effect of the type of endodontic sealer on the bond strength between fiber post and root wall dentin. J Dent 2014;11(4):455-463.

12. Ruiz L, Gomes GM, et al. Effect of root canal sealers on bond strength of fiber posts to root dentin cemented after one week or six months. Iran Endod J 2018;13(1):54-60. DOI: 10.22037/iej.v13i1.17998.

13. Iglesia-Puig MA, Arellano-Cabornero A. Fiber-reinforced post and core adapted to a previous metal ceramic crown. J Prosthet Dent 2004;91(2):191-194. DOI: 10.1016/S0022391303007777.

14. Capar ID, Aydinbelge HA. Surface change of root canal dentin after the use of irrigation activation protocols: electron microscopy and an energy-dispersive X-ray microanalysis. Microsc Res Tech 2013;76(9):893-896. DOI: 10.1002/jemt.22244.

15. Aleisa $\mathrm{K}, \mathrm{Al}$-Dwairi $\mathrm{ZN}$, et al. In vitro evaluation of the effect of different endodontic sealers on retentive strength of fiber posts. Oper Dent 2013;38(5):539-544. DOI: 10.2341/12-414-L.

16. Goracci C, Ferrai M. Current perspectives on post systems: a literature review. Aust Dent J 2011;56(1):77-83. DOI: 10.1111/j.18347819.2010.01298.x.

17. Zhou L, Wang Q. Comparison of fracture resistance between cast posts and fiber posts: a meta-analysis of literature. J Endod 2013;39(1): 11-15. DOI: 10.1016/j.joen.2012.09.026.

18. Cohen BI, Volovich Y, et al. The effects of eugenol and epoxy-resin on the strength of a hybrid composite resin. J Endod 2002;28(2):79-82. DOI: 10.1097/00004770-200202000-00007.

19. Lima AC, Rached-Junior FJ, et al. Influence of sealer and lightcuring units on push-out bond strength of composite resin to weakened roots. Braz Dent J 2016;27(4):430-435. DOI: 10.1590/01036440201601010.

20. Kasam S, Mariswamy AB. Efficacy of different methods for removing root canal filling material in retreatment - an in vitro 
study. J Clin Diagn Res 2016;10(6):6-10. DOI: 10.7860/ JCDR/2016/17395.7904.

21. Horvath SD, Altenburger MJ, et al. Cleanliness of dentinal tubules following gutta-percha removal with and without solvents: a scanning electron microscopic study. Int Endod J 2009;42(11):10321038. DOI: 10.1111/j.1365-2591.2009.01616.x.

22. Altmann ASP, Leitune VCB, et al. Influence of eugenol-based sealers on push-out bond strength of fiber post luted with resin cement: systematic review and meta-analysis. J Endod 2015;41(9):1418-1423. DOI: 10.1016/j.joen.2015.05.014.
23. Bronzato JD, Cecchin D, et al. Effect of cleaning methods on bond strength of self-etching adhesive to dentin. J Conserv Dent 2016;19(1): 26-30. DOI: 10.4103/0972-0707.173189.

24. Bramante CM, Fidelis NS, et al. Heat release, time required, and cleaning ability of two $\mathrm{R}$ and protaper universal retreatment systems in the removal of filling material. J Endod 2010;36(11):1870-1873. DOI: 10.1016/j.joen.2010.08.013.

25. Kuga MC, Faria G, et al. Persistence of epoxy-based sealer residues in dentin treated with different chemical removal protocols. Scanning 2012;35(1):17-21. DOI: 10.1002/sca.21030. 